\title{
Odor-intensity interaction in binary and ternary mixtures
}

\author{
BIRGITTA BERGLUND and MATS J. OLSSON \\ Karolinska Institute, Stockholm, Sweden \\ and Stockholm University, Stockholm, Sweden
}

\begin{abstract}
Eight subjects scaled the overall perceived odor intensity of binary and ternary mixtures of the odorous substances pyridine, acetone, and ethyl acrylate. The results concerning basic principles of additivity for binary mixtures comply with those of earlier studies. For the ternary mixtures, the degree of arithmetic additivity in odor intensity seemed lower when a third component was added to a binary mixture than when the second component was added to a single odor, but this did not reach statistical significance. In general, the degree of arithmetic additivity inherent in the power function for single substances was found to be of the same size as the degree of additivity for binary as well as ternary mixtures. However, for binary mixtures, the additivity of the mixtures was found to approximate that of the substance with the lower exponent. In addition, the degree of additivity of the binary mixture was monotonically related to the average arithmetic additivity of the power functions for the two components.
\end{abstract}

In olfaction, the knowledge accumulated for how two odorous substances combine to a new percept is quite extensive but less so for more complex mixtures. The binary mixture percept $\left(\Psi_{i j}\right)$ is usually related either to the percepts of the two single components when the components are presented singly $\left(\Psi_{i}\right.$ and $\left.\Psi_{j}\right)$, the perceptual approach, or to the concentrations of the single components ( $\Phi_{i}$ and $\Phi_{j}$ ), the psychophysical approach. The psychophysical approach was mainly employed in the early studies in mixture perception (Baker, 1963, 1964; Kendall \& Neilson, 1966; Köster, 1969; Köster \& MacLeod, 1975; Rosen, Peter, \& Middleton, 1962), whereas the perceptual approach predominated in later research (Berglund, 1974; Berglund, Berglund, \& Lindvall, 1971; Berglund, Berglund, Lindvall, \& Svensson, 1973; Berglund \& Olsson, 1993; Cain, 1975; Cain \& Drexler, 1974; de Wijk, 1989; Gregson, 1980, 1983, 1986; Jones \& Woskow, 1964; Laffort \& Dravnieks, 1982; Laing \& Francis, 1989; Laing, Panhuber, Willcox, \& Pittman, 1984; Laing \& Willcox, 1983, 1987; Moskowitz \& Barbe, 1977). Another important distinction in research strategy is whether the components are distinctly perceivable (heterogeneous) or not (homogeneous) inside the mixture. Consequently, the perceptions of the component odors when these odors are presented alone have either been compared to the perception of the odor components inside the mixtures $\left(\Psi_{i}^{\prime}, \Psi_{j}^{\prime}\right.$; e.g., Laing et al., 1984) or to the overall perception of

This research was sponsored by grants from the Swedish Council for Research in the Humanities and Social Sciences and the Swedish Council for Building Research. Thanks go to T. Rehn and B. Sandin for experimental assistance. The authors are affiliated with the Institute of Environmental Medicine at the Karolinska Institute, as well as the Department of Psychology at Stockholm University. Correspondence should be addressed to B. Berglund, Department of Psychology, Stockholm University, S-106 91, Stockholm, Sweden. the mixture $\left(\Psi_{i j}\right.$; see also Berglund \& Olsson, 1993; Laffort, 1989).

Most of the empirical research has dealt with the overall perceived intensity of mixtures, rather than with perceived quality or hedonic tone, and so forth. For binary mixtures, the findings may be summarized as follows: (1) Hypoaddition prevails for odor intensity; the overall mixture intensity is always weaker than the arithmetic sum $\left(\boldsymbol{\Psi}_{i j}<\boldsymbol{\Psi}_{i}+\boldsymbol{\Psi}_{j}\right)$ and always stronger than the arithmetic mean $\left[\Psi_{i j}>\left(\Psi_{i}+\Psi_{j}\right) / 2\right]$ of the separately presented component odor intensities. It follows that the mixture intensity is never weaker than the weakest component $\left[\boldsymbol{\Psi}_{i j}\right\rangle$ $\min \left(\Psi_{i}, \Psi_{j}\right)$ ]. (2) Most types of mixtures hitherto studied yield compromise $\left(\boldsymbol{\Psi}_{i}<\boldsymbol{\Psi}_{i j}<\boldsymbol{\Psi}_{j}\right)$ to some extent. (3) Other findings less agreed upon include a level dependency as well as an asymmetry in mixture summation. Level independency means that the odor summation process does not differ between weak and strong odors, whereas asymmetry suggests that a strong odor of substance $i$ and a weak odor of substance $j$ would result in an odor of different intensity in comparison with the opposite case.

Only a few studies of mixtures have addressed the question of odor-intensity interaction for more complex mixtures-that is, for three or more component odors. This is true for both the psychophysical approach (Baker, 1963; Kendall \& Neilson, 1966; Moskowitz \& Barbe, 1977) and the perceptual approach (Berglund, 1974; Laffort \& Dravnieks, 1982; Laing et al., 1984). Others have focused on, for example, perceived complexity of complex mixtures (Jellinek \& Köster, 1979, 1983; Laing \& Francis, 1989). The complex-mixture experiments are cumbersome and, consequently, little is known about the odor summation process for these mixtures. However, Berglund (1974) showed that for mixtures of equally in- 
tense odors, up to the tested five-odor ones, hypoaddition prevails and there is clearly no level dependency. Interestingly, the odor intensity of the mixture relative to the component, when equally intense components are added, is only slightly more intense than that of one component presented alone. In other words, a strong attenuation of input occurs for complex mixtures.

Our aim in the present experiment was to examine the summation process for perceived odor intensity of single odors and their binary and ternary mixtures. Particularly, the intensities of the three components were compared both with the overall intensity of the three binary mixtures created from these components and with the overall intensity of the ternary mixture. Thus, a perceptual approach was utilized and homogeneous mixture percepts were assumed. Specifically, some of the working principles for binary odor summation were scrutinized, such as hypoaddition, compromise, level dependency, and symmetry. In addition, the degree of arithmetic additivity was studied for single substances as well as binary and ternary mixtures, and in doing so an attempt was made to compare mixture additivity with the growth reflected in the exponents of the psychophysical power function for single substances.

\section{METHOD}

\section{Subjects}

Eight subjects, 1 woman and 7 men, took part in the main experiment. They were between 18 and 33 years old and only 1 was a smoker. Our subjects were selected from a larger group who took part in a preliminary experiment. None of the subjects had any previous experience in olfactory experiments, and they were unaware of the purpose and conditions of the experiment. Another group of 4 subjects took part in a preliminary experiment used for selecting the appropriate stimulus concentrations.

\section{Equipment}

The odorants were presented in an odor laboratory described elsewhere (Lindvall, 1970). It consists essentially of a ventilated and air-conditioned test chamber with an odor exposure hood connected to a dynamic olfactometer installed in a separately vented closed hood in an adjacent laboratory room. The hood provided a tight fit around the seated subject's face, allowing fairly natural breathing. The concentrations presented to the subjects were controlled by the olfactometer utilizing steel capillaries (different length and bore) calibrated for flow and pressure. The vented air in the housing for the equipment, test chamber, and the waiting room for the subjects was filtered for particles and other odorous contaminations and kept at a constant temperature and humidity. The test chamber as well as the exposure hood were kept at partial overpressures to keep out extraneous odors.

\section{Stimuli}

The three odorous substances presented in the experiment were ethyl acrylate, pyridine, and acetone. These were chosen in order to represent both pleasant and unpleasant odors as well as odorous substances with different exponents in the psychophysical power function (Equation 1). The latter refers to the growth rate with concentration for the single substance that may be suspected to be associated with the additivity reflected in odor mixtures. Both acetone and pyridine are malodorous substances. Acetone is a solvent that may be perceived as pungent, and pyridine can serve as an irritant at higher concentrations. Ethyl acrylate is the most pleasant odor of the three and it is known to have a somewhat low exponent of the power function.

In a preliminary experiment involving 4 subjects, the perceived odor intensities of the three odorous substances were judged with the method of magnitude estimation. The results were then used for selecting the seven concentrations of each odorous substance for the main experiment so that they would be equally intense at seven different perceived intensity levels. In Table 1 , the seven concentrations (ppm) are shown (left part) together with their perceived odor intensities (right part) obtained in the main experiment.

The seven concentrations of the three odorous substances were combined into three sets each of 17 binary mixtures selected from a square matrix according to Table 2. Furthermore, the ternary mixtures were formed by combining the seven concentration numbers, $1-1-1,2-2-2$, and so on to $7-7-7$, representing the different perceived intensity levels that in the selection of stimuli were intended to be equally intense (cf. Table 1). A "blank" produced from charcoal-filtered air was also added to the set of stimuli. In summary, the subjects were presented with 80 different stimulus presentations: 21 concentrations of single substances, 51 binary mixtures, 7 ternary mixtures, and 1 blank.

\section{Procedure}

In the main experiment, the perceived odor intensity was obtained through cross-modality matching by using a finger-span technique that was successful earlier in adaptation experiments by Ekman,

Table 1

The Seven Concentrations of Ethyl Acrylate, Pyridine, and Acetone Used in the Main Experiment (Left), Displayed Together With the Perceived Odor Intensities of the Single Substances, the Binary Mixtures, and the Ternary Mixtures Determined in the Main Experiment

\begin{tabular}{|c|c|c|c|c|c|c|c|c|c|c|}
\hline \multirow[b]{3}{*}{ No. } & \multicolumn{3}{|c|}{ Concentration (ppm) } & \multicolumn{7}{|c|}{ Perceived Intensity (ME) } \\
\hline & \multicolumn{3}{|c|}{ Single } & \multicolumn{3}{|c|}{ Single } & \multicolumn{3}{|c|}{ Binary } & \multirow{2}{*}{$\frac{\text { Ternary }}{\text { EPA }}$} \\
\hline & $\mathrm{E}$ & $\mathbf{p}$ & $A$ & $E$ & $\mathbf{P}$ & A & EP & $\overline{\text { EA }}$ & PA & \\
\hline 1 & .000353 & 0.025 & 95.8 & 12.75 & 5.30 & 9.6 & 8.74 & 18.77 & 16 & 12.85 \\
\hline 2 & .001434 & 0.051 & 139.6 & 12.18 & 10.72 & 11 & 13.29 & 19.24 & & 15.03 \\
\hline 3 & 0.005622 & 0.102 & 213.1 & 18.71 & 7.78 & 16.96 & 18.20 & 29.54 & 19. & 21.87 \\
\hline 4 & 0.02295 & 0.220 & 303.1 & 35.72 & 14.84 & 24.37 & 34.93 & 33.34 & 19.63 & 36.12 \\
\hline 5 & 0.0975 & 0.487 & 499.9 & 33.51 & 20.13 & 28.84 & 34.93 & 40.06 & 42.30 & 48.29 \\
\hline 6 & 0.367 & 0.983 & 690.3 & 53.83 & 34.87 & 34.55 & 63.00 & 60.84 & 51.54 & 69.31 \\
\hline 7 & 1.581 & 1.977 & $1,040.4$ & 73.66 & 56.49 & 47.66 & 74.06 & 71.73 & 60.06 & 70.54 \\
\hline
\end{tabular}

Note-ME, magnitude estimation; $E$, ethyl acrylate; $P$, pyridine; $A$, acetone. EP refers to the mixture of $E$ and $P$ and so on. EPA refers to a mixture of $E, P$, and $A$ 
Table 2

The Concentration Combinations Selected for the Three Binary Mixtures in the Main Experiment

\begin{tabular}{cccccccccc}
\hline & \multicolumn{7}{c}{ Substance $j$} & & \\
Substance $i$ & 0 & 1 & 2 & 3 & 4 & 5 & 6 & 7 \\
\hline 0 & $0 / 0$ & $0 / 1$ & $0 / 2$ & $0 / 3$ & $0 / 4$ & $0 / 5$ & $0 / 6$ & $0 / 7$ \\
1 & $1 / 0$ & $1 / 1$ & & & & $1 / 5$ & & $1 / 7$ \\
2 & $2 / 0$ & & $2 / 2$ & & $2 / 4$ & & & \\
3 & $3 / 0$ & & & $3 / 3$ & & & & $3 / 7$ \\
4 & $4 / 0$ & & $4 / 2$ & & $4 / 4$ & & $4 / 6$ & \\
5 & $5 / 0$ & $5 / 1$ & & & & $5 / 5$ & & \\
6 & $6 / 0$ & & & & $6 / 4$ & & $6 / 6$ & \\
7 & $7 / 0$ & $7 / 1$ & & $7 / 3$ & & & & $7 / 7$ \\
\hline
\end{tabular}

Note-Numerals represent concentration numbers.

Berglund, Berglund, and Lindvall (1967). The finger span was set by the subject so as to be perceived as equal to the odor intensity. Following each match, the subject again placed the index finger at the position of the original maximum finger span. After the main experiment with the odorants, the finger-span data were transformed individually into perceptual measures by scaling of the span. The method of magnitude production was then used, whereby each subject produced, in random order, $5 \%, 15 \%, 25 \%, 35 \%, 45 \%, 55 \%$, $65 \%, 75 \%, 85 \%$, and $95 \%$ of his/her maximum subjective span. Each adjustment was made 10 times by each subject in random order, always without visual cues. This scaling procedure was used in order to counteract the tendency of subjects to generate a disproportional amount of manageable numbers, which would easily distort the mean. For the odor presentations, the subject was instructed to put his/her face up against the exposure hood. Upon a signal from a light mounted inside the hood, the subject was to inhale once. The interstimulus interval was kept between 30 and $60 \mathrm{sec}$, depending on the time required to adjust the finger span to the perceived intensity of the odor. Each subject was presented with one unique random order of the whole set of 240 stimulus presentations, which included three repeated presentations of each stimulus. The presentations were given in 15 blocks of 16 , with a 15 -min rest between blocks and a longer break for lunch after 10 blocks. In this way, each subject was available for the experiment during approximately $6 \mathrm{~h}$ of one day. Before participating in the morning as well as after the lunch break, the subject was conditioned $20 \mathrm{~min}$ to the background of purified air in the waiting room. In the morning the subjects took part in a training session, which was followed by the main experiment.

\section{RESULTS AND DISCUSSION}

\section{Odor-Intensity Scales}

For each subject, each individual finger-span matching was transformed into perceptual values with the aid of the psychophysical functions determined for finger span after the experiment. The individual odor-intensity scales were constructed from the arithmetic means of the three odorintensity values for each stimulus. Excluding the presentations of the blank, the whole data set contained 3\% zero responses to odor presentations, which is acceptable. The zeros were excluded in the calculations. This is not a selfevident choice, but their presence, or absence, in the data does not affect the main conclusions. The group scale of odor intensity was determined as the arithmetic mean values of the individual scales based on the 80 stimuli representing single odorants, the blank, the binary mixtures, and the ternary mixtures. The scales for the single odorants and the ternary mixtures are shown in Table 1.

The perceived odor intensity plotted as a function of concentration is most commonly represented by $\mathrm{S}$. S. Stevens's power function (see, e.g., Engen, 1982),

$$
\Psi=c \Phi^{n},
$$

where $\Psi$ is the perceived odor intensity, $\Phi$ is the concentration, and $c$ and $n$ are the multiplicative constant and exponent, respectively. As shown in Figure 1 in logarithmic coordinates, power functions fit well for the three odorous substances scaled. As expected in selecting the odorous substances for this mixture experiment, the exponents are higher for pyridine $(n=0.51, c=34.1)$ and acetone $(n=0.67, c=0.463)$ than for ethyl acrylate $(n=0.23, c=68.3$ ).

In the following text, some principles of odor summation are studied for the binary and ternary mixtures. Specifically, the data are analyzed with regard to degree of arithmetic additivity, compromise, level dependency, and symmetry. In order to stress the fact that the perceived odor intensity is operationally defined by the transformed finger-span responses, different symbols are used to denote the perceived intensity $(\boldsymbol{\Psi})$ and the subjects response $(\mathrm{R})$.

\section{Binary Mixtures}

Degree of arithmetic additivity. The additivity in binary mixture summation may be elucidated by two principles expressed mathematically as ratios (Patte \& Laffort, 1979). The first ratio (o), called the degree of arithmetic additivity, compares the perceived intensity of the mixture with the sum of the perceived intensities of its components:

$$
\sigma=\boldsymbol{\Psi}_{i j} /\left(\boldsymbol{\Psi}_{i}+\boldsymbol{\Psi}_{j}\right)
$$

The other ratio $(\tau)$, the component intensity proportion, denotes the intensity of one component as a proportion of the sum of the component intensities:

$$
\tau=\Psi_{i} /\left(\Psi_{i}+\Psi_{j}\right) .
$$

The indices $i, j$, and $i j$ represent the one substance, the other, and the mixture, respectively. By plotting the $\sigma$ values as a function of the $\tau$ values, the effect on the degree of arithmetic additivity of the component intensity proportion is visualized. As can be seen in Figure 2, the degree of additivity tends to increase as the component intensites grow unequal; at least this trend is clear for the binary mixture of ethyl acrylate and pyridine (left diagram). As a rule, the mixtures are always perceived to be stronger than the arithmetic mean and weaker than the sum of the component intensities. There are only two exceptions to this rule (see Figure 2), which may be disregarded because of the extreme contribution from 1 subject in one of the cases and a statistically insignificant deviation in the other. A rather large scatter of data points is found for all three binary mixtures, especially for the 


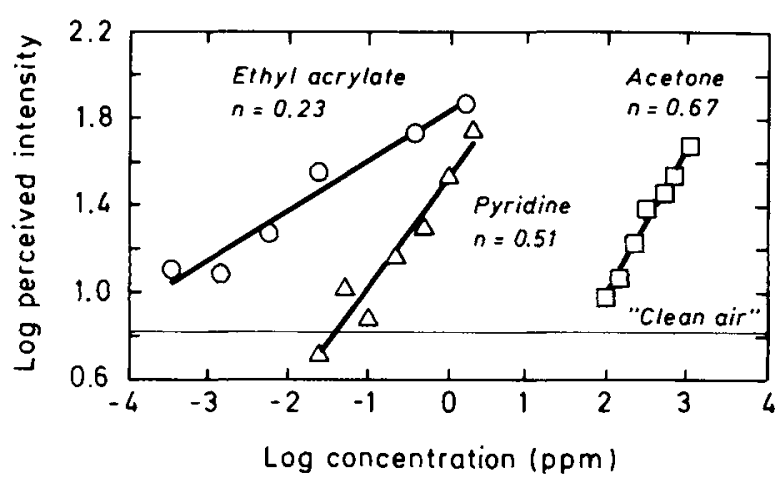

Figure 1. Psychophysical power functions for ethyl acrylate, pyridine, and acetone, obtained in the main experiment. The thin horizontal line at the bottom denotes the odor intensity of the blank presentation (clean air). The exponent of the power function is denoted $n$ (Equation 1).

two that contain acetone. This is not unusual for such plots, because the error variance of measurement is easily magnified in the ratio calculations according to Equations 2 and 3 (see Berglund \& Olsson, 1993).

Level dependency. One issue addressed by a few researchers is the existence of level dependency in the summation process for mixtures (Berglund, 1974; Berglund \& Olsson, 1993; Laing et al., 1984). That is, do two weak odors in a mixture yield the same degree of additivity $(\sigma)$ as two strong odors? From the whole data set of both individual scales and group scales, a subset of the equally intense component odors entering a binary mixture was created. Since we did not succeed perfectly in the original choices of concentrations matched for odor intensity (see Table 1), only the binary cases fulfilling the following criterion for equality were used: $0.8<\left(R_{i} / R_{j}\right)<1.25$. In Figure 3 , the perceived odor intensity of the mixtures is plotted against the mean component odor intensities for the cases of equally intense components. The regression lines fitted to the data points are forced through the origins (filled circles represent group data; open circles represent individual data). Except in the case of the binary mixture of pyridine and ethyl acrylate, the scatter of data points is quite large but they approximate linearity. The three binary mixtures show no pronounced level dependency. In comparison with earlier published figures of six different types of mixtures, of which none show level dependency (Berglund, 1974; Berglund \& Olsson, 1993), the data points fall somewhat above the line forced through the origin for weaker odors. If regression lines with intercepts were drawn in Figure 3, all would have positive intercepts. However, only the intercept of the pyridineacetone mixture would actually reach a statistically significant deviation from zero, as is revealed by a $95 \%$ confidence interval for the intercept (two-tailed). Before this tendency is interpreted as being related to the particular binary mixtures, a possible association with the fingerspan matching method used in this experiment should be ruled out. Compared to the method of magnitude estimation used in the earlier studies, there is an extra transformation step from the physical finger spans into perceptual spans. However, the slopes of the lines in Figure 3 are of the same size as the slopes representing additivity for equally intense odors in other binary mixtures (cf. Berglund, 1974; Berglund, Berglund, \& Lindvall, 1976).

Symmetry. Another principle of interest in odor summation is the symmetry (or asymmetry) in mixture data. The question is whether the degree of additivity is as high for a strong odor of substance $i$ mixed with a weak odor of substance $j$ as for the opposite case. This issue was recently addressed by Berglund and Olsson (1993) for a binary mixture of pyridine and dimethyl disulfide that showed a slight asymmetry. Unfortunately, from the present data for three binary mixtures it is not possible

\section{Ethyl acrylate-Pyridine}

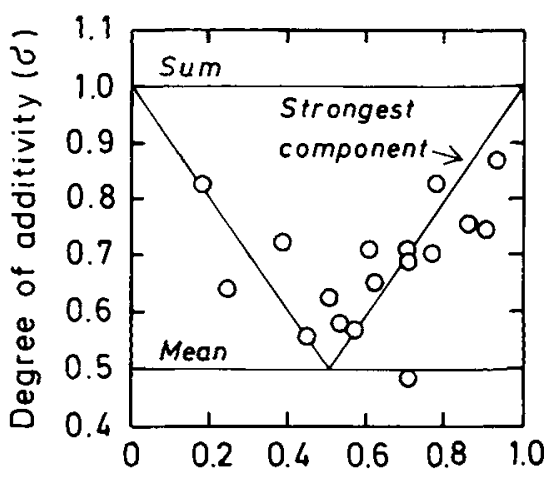

Ethyl acrylate -Acetone

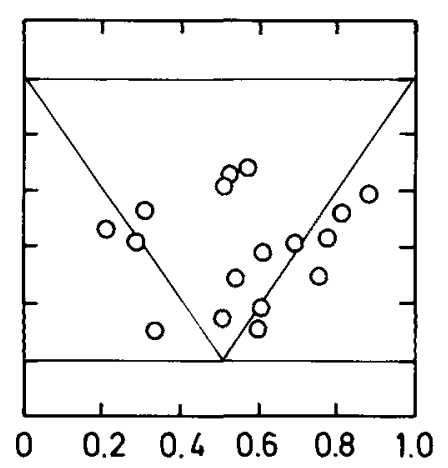

Pyridine - Acetone

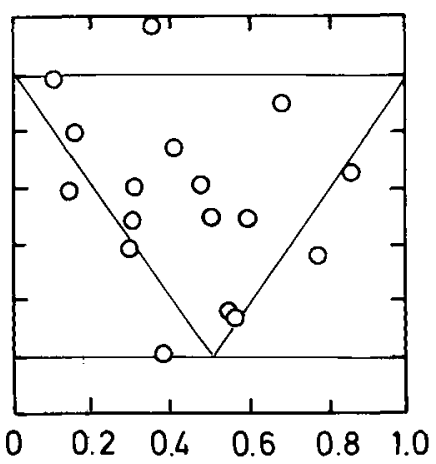

Component intensity proportion $(T)$

Figure 2. The degree of arithmetic additivity $(\sigma)$ in the binary mixtures is displayed in relation to the component intensity proportion $(\tau)$. The outcomes for three simple summation models are given: the arithmetic sum (the thin horizontal line at the top), the arithmethic mean (the horizontal line at the bottom), and the strongest component model (the V-shaped curve). 

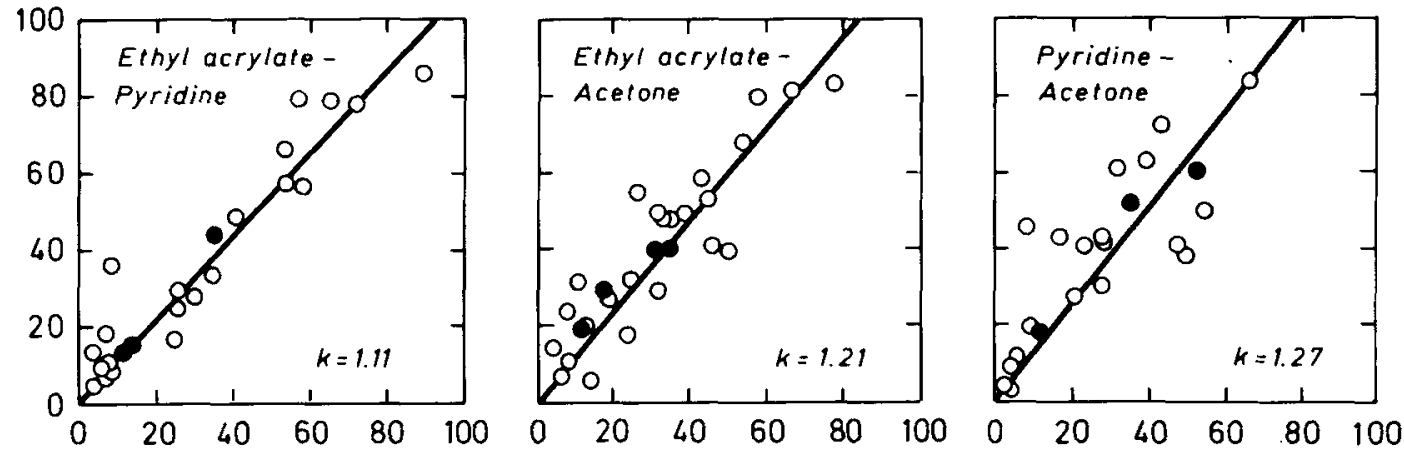

Mean perceived intensity of equally intense odor components

Figure 3. The perceived odor intensity of the mixtures $\left(\boldsymbol{R}_{i j}\right)$ is plotted against the arithmetic mean of the equally intense component odors $\left(\boldsymbol{R}_{i}, \boldsymbol{R}_{j}\right)$. The regression lines fitted to the data points were forced through the origins. The filled circles represent group data, and the open circles represent individual data. The slopes are denoted $k$.

to draw any conclusion regarding the symmetry-asymmetry issue (see Figure 2).

Compromise. Compromise is a quite characteristic phenomenon for binary odor mixtures (Berglund et al., 1976; Cain, 1975). As noted above, the term is used for the case in which the odor intensity of the mixtures falls between the intensities of the component odors $\left(\Psi_{i}<\right.$ $\left.\Psi_{i j}<\Psi_{j}\right)$. That is, arithmetic addition of odor intensities results in perceptual hypoaddition for the intensity ranges represented by the two components. For the 51 binary mixtures tested in this study, almost one half (41\%) showed compromise, whereas the others showed hypoaddition in the form of $\Psi_{i j}<\Psi_{i}+\Psi_{j}$.

The curve marked "strongest component" in the three panels of Figure 2 represents the theoretical outcome for the strongest component model:

$$
\Psi_{i j}=\max \left(\Psi_{i}, \Psi_{j}\right) \text {. }
$$

All the data points that fall below this $V$-shaped curve representing the strongest component model are binary mixtures representing the phenomenon of compromise.

\section{Exponents and Mixture Additivity}

The three odors used in this experiment of course differ in many respects, physically as well as perceptually. One difference occurs in the exponent $(n)$ of the psychophysical power function, reflecting growth or "self-addition" (Figure 1). Two of the odorous substances yield a relatively high exponent (pyridine, $n=0.51$; acetone, $n=0.67$ ), and the third, a low one (ethyl acrylate, $n=0.23$ ). Laffort (1989) derived a relationship between the degree of arithmetic additivity $(\sigma)$ for odor intensities of single substances and the component intensity proportion $(\tau)$, using the exponent as a parameter:

$$
\sigma=\left[\tau^{1 / n}+(1-\tau)^{1 / n}\right]^{n}
$$

or, rewritten for the case in which two identical perceptual intensities (as well as physical concentrations) of a single substance are added (i.e., self-addition):

$$
\sigma=0.5 * 2^{n} \quad\left(\tau=0.5 ; \Psi_{i}=\Psi_{i}\right)
$$

If Equation 6 is applied to the exponents of the odorous substances, the additivity in terms of self-addition is $\sigma=$ 0.71 for pyridine, $\sigma=0.79$ for acetone, and $\sigma=0.59$ for ethyl acrylate. These three estimates of the degree of additivity based on the exponents agree well with the degree of additivity found for both the binary and the ternary mixtures. As can be seen from Figure 2, most of the $\sigma$ values fall within this range $(0.55-0.80)$.

Interestingly, as can be seen in Figure 4, the additivity of the mixtures seems to approximate that of the substance with the lower exponent. In the figure, the additivity index for the single component is calculated from the exponents according to Equation 6-that is, for the case in which the components are equally strong. The additivity index for the binary mixture is derived from the slopes $(k)$ in Figure 3, which also apply only to equal cases. The additivity index for the mixture is estimated as $\sigma=k / 2$.

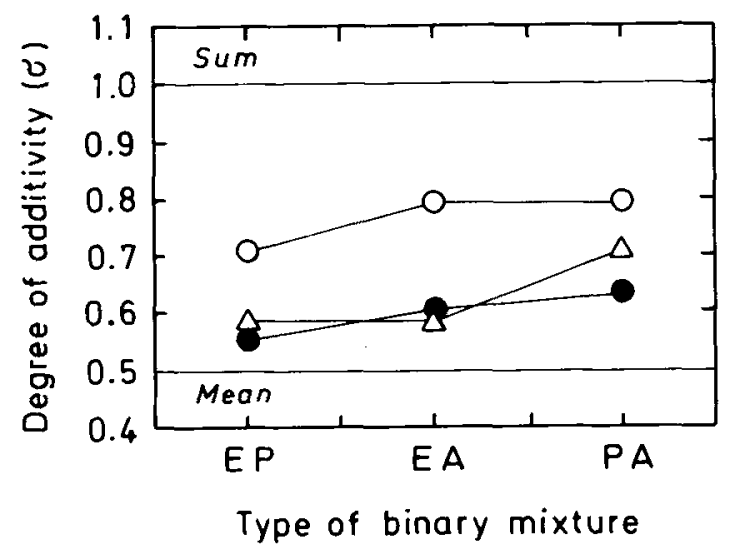

Figure 4. The additivity index $(\sigma)$ for the binary mixture (filled circles) is compared with the self-additivity indices of its two components (the more additive component is symbolized by open circles and the less additive by open triangles). The additivity index for the single component is calculated from the exponents according to Equation 6-that is, for the case in which the components are equally strong. The additivity index for the binary mixture is derived from the slopes $(k)$ in Figure 3, which also apply only to equal cases. The additivity index for the mixture is estimated as $\sigma=k / 2$. 
Another interesting observation in Figure 4 is that the additivity of the mixture keeps rank order with the general (average) additivity of the two components involved. This sameness in additivity values should, however, not lead us to conclude that the summation process in mixtures and in single odors is the same. This hypothesis has been addressed before and found to be questionable (Berglund \& Olsson, 1993; cf., however, Bartoshuk, 1975, and Frijters \& Oude Ophuis, 1983, on taste mixtures) on the grounds that the odor summation process in mixtures can show asymmetry and compromise. These two principles are not inherent in the psychophysical power function.

\section{Ternary Mixtures}

In order to show the degree of arithmetic additivity for binary mixtures appropriately, it is imperative to compensate for the variance in the component intensity proportion ( $\tau$ in Equation 3). In dealing with ternary mixtures, there is no simple illustration of the degree of additivity like that obtained for binary mixtures in Figure 2 . This problem has been addressed before by Berglund (1974). For a ternary mixture of pyridine, hydrogen sulfide, and dimethyl disulfide, she successfully confirmed that the odor intensity of 290 mixtures of one ternary substance combination can be predicted from information on how the three odors mix according to pairs. This was done with the aid of a vector model originally described by Berglund et al. (1973). In the generalized model for complex mixtures, Berglund (1974) assumed the following: when two odors are mixed, a certain "area" of interaction is activated, and when a third odor is added to the two-odor mixture, two new "areas" of interaction are made active, as well as a common "area" unique to the three. However, her data on four and five odor mixtures (but not three odor mixtures) showed that a slightly different view only allowing for one common interaction "area" for all components in the mixture may be a better choice. This would imply that the degree of interaction is less than the one based on the three pairwise overlapping interaction "areas" and the one common (for the three). However, the present experiment involved only seven different intensity combinations of the ternary mixture, which is not enough for a meaningful test.

One interesting possibility for assessing the additivity in the present data is to simply regard the ternary mixture as composed of one binary mixture combined with a single odorous substance. According to this approach, three cases will emerge from every ternary mixturethat is, $R_{i j k}$ is a function of the binary mixture $R_{i j}$ and the single odor $R_{k}$, of $R_{i k}$ and $R_{j}$, and of $R_{j k}$ and $R_{i}$. From the seven ternary mixtures in the present experiment, 21 such pairs of "components" may be formed.

Now it is possible to compare the 21 combinations of the two "components" (i.e., binary mixture plus single odor) with all the binary mixtures involved in the ternary mixtures (cf. Table 1). The comparison is made by analyzing the relationship between the degree of arithmetic additivity ( $\sigma$ in Equation 2) and the component intensity proportion ( $\tau$ in Equation 3). The analysis gives an answer to the following question: Is there a difference between adding a second component odor to a single odor versus adding a third component to a two-component mixture? A visual inspection of Figure 5 suggests that the degree of arithmetic additivity for the binary mixtures is somewhat higher than that for the particular tested case of ternary mixtures (i.e., a third component is added to a binary mixture). Here, the two cases of 21 combinations for binary mixtures (upper diagram) and binary mixtures plus a single component (lower diagram) are in the histograms of $\sigma$ values representing degree of arithmetic additivity. The difference in mean additivity index for each subject $(n=8)$ between the two different types of mixtures was put to test (paired two-group $t$ test). The test showed no significant difference in additivity between binary and ternary mixtures. The two sets of $\sigma$ values tested both rely on approximately the same range of $\tau$ values. This can be seen in Figure 6, in which the open circles denote the 21 binary cases, and the open triangles, the ternary cases.

In Figure 6, the cases of compromise appear as data points below the $\mathrm{V}$-shaped curve representing the stron-

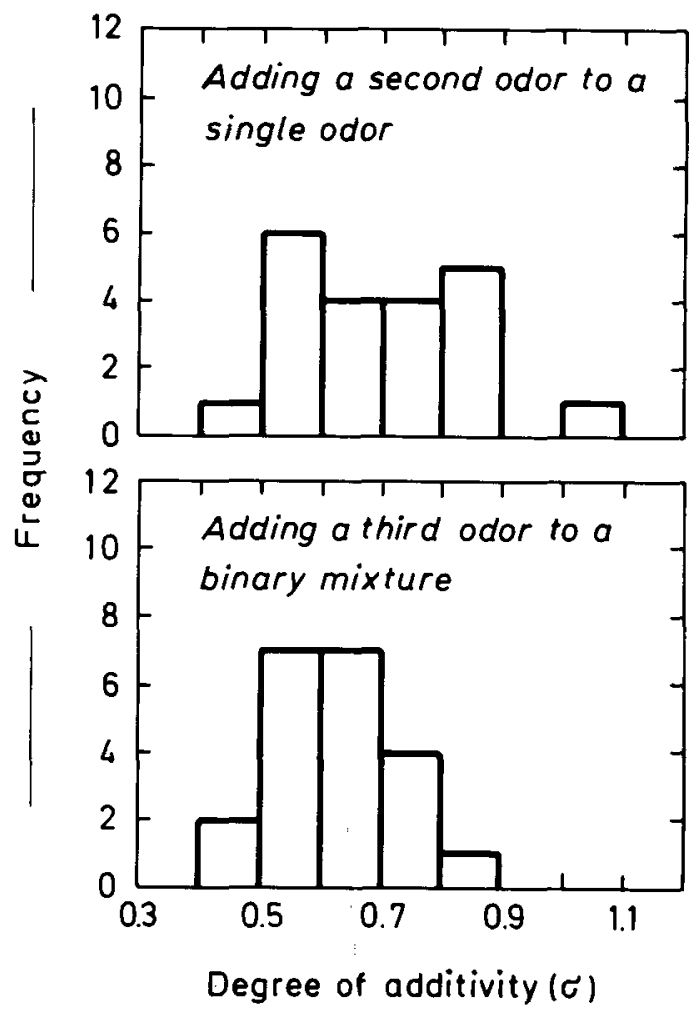

Figure 5.The upper diagram shows the frequency distribution of $\sigma$ values $\left[\boldsymbol{R}_{i j} /\left(\boldsymbol{R}_{I}+\boldsymbol{R}_{j}\right)\right]$, which denote the degree of additivity in mixtures, for the case in which a second component is added to a first. The lower diagram shows the frequency distribution of $\sigma$ values $\left[R_{i j k} /\left(R_{i j}+R_{k}\right)\right]$ for the case in which a third component is added to a binary mixture. 
O BINARY

$\triangle$ TERNARY

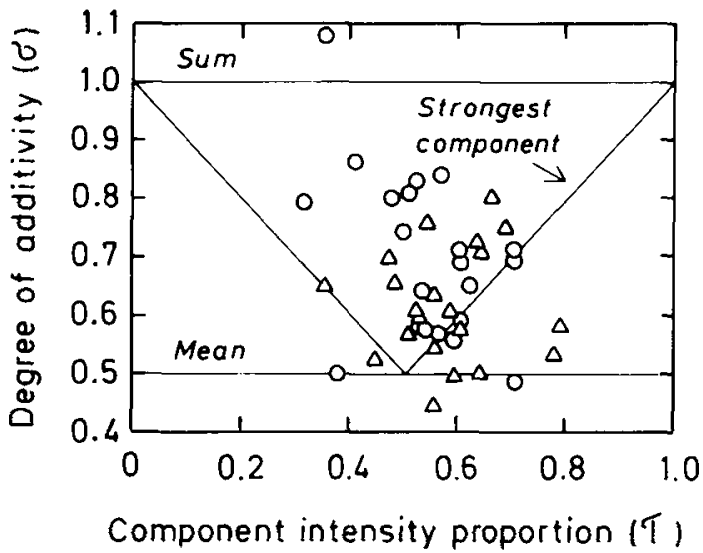

Figure 6.The degree of arithmetic additivity $(\sigma)$ in binary (open circles) and ternary (open triangles) odor mixtures is displayed in relation to the component intensity proportion ( $\tau$ ). The ternary mixtures are combinations of a single odorant and a binary mixture [i.e., $\left.\sigma=R_{i j k} /\left(R_{i j}+R_{k}\right)\right]$. Outcomes for three simple summation models are given: the arithmetic sum (the thin horizontal line at the top), the arithmethic mean (the horizontal line at the bottom), and the strongest component model (the $V$-shaped curve).

gest component model. It is obvious that neither the ternary mixtures nor the binary mixtures show any compromise for "component" odors of equal intensity defined as $0.44<\tau<0.56$.

In the present experiment, there were enough equalintensity cases to allow analysis of the binary mixtures (Figure 2), but only one of the seven ternary mixtures in the group data fulfills our requirement for equality (the mixture of Concentration No. 2; Table 1). For this equalintensity case, the ternary mixture is approximately 1.30 times stronger than the mean of its single components when presented alone. This factor of additivity corresponds well with the one that Berglund (1974) found for the ternary mixture of hydrogen disulfide, pyridine, and dimethyl disulphide (1.31). Therefore, it seems as if a strong attenuation applies to ternary mixtures as well as binary mixtures. Moreover, Berglund showed that the additivity actually decreased when a fourth and fifth equally strong component were added to the ternary mixture (1.12 and 1.09 , respectively), suggesting that it is not only possible but in fact a general rule that the overall intensity of a mixture decreases with the addition of another odor. It should be pointed out here that these findings must not be confused with the occurrence of compromise for binary and ternary mixtures, for two reasons: First, compromise occurs quite often, as we have seen, but it is not the general rule for the additivity of binary and ternary mixtures. Second, compromise occurs generally for mixtures of unequally strong components.

\section{CONCLUSIONS}

The perceptual arithmetic of odors seems to give a good picture of some basic principles of how odors add to each other. From this perspective, the odor summation process shows a strong attenuation for binary and ternary mixtures as well as single odors. That is, the attenuation that we can observe when more of the same substance is added, or, put in another way, the extent to which the psychophysical function is compressed, has, on the whole, the same magnitude as the attenuation that we can observe when we add one odor to another, or a third odor to a binary mixture. However, if we regard the criterion for complete additivity as indicated by the exponents, one might not speak of attenuation at all.

Specifically, the data may be summarized as follows:

1. Odor mixtures generally, if not always, yield hypoaddition as shown in this study as well as in several other studies.

2. An equally well-established fact is that the odor mixture intensity always exceeds the mean of its component intensities.

3. Compromise is common, indicating a pronounced attenuation in the odor summation process different from that found for self-addition.

4. The idea that the odor summation process is level dependent as a general rule is rejected.

5 . When the additivity of a binary mixture is compared with the additivity indicated by the specific power function that describes the psychophysical relationship for each component, the mixture additivity seems to be of the same size as the self-additivity of the one with the smallest exponent.

6 . The additivity of the mixture keeps rank order with the average additivity of the single odors that constitute the mixture.

7. For ternary mixtures, the additivity seems less than that for binary mixtures. But no statistically significant difference was observed, and therefore the additivity of binary mixtures and that of ternary mixtures are regarded to be of the same size.

\section{REFERENCES}

BAKER, R. A. (1963). Odor effects of aqueous mixtures of organic chemicals. Journal of the Water Pollution Control Federation, 35, 728-741.

BAKER, R. A. (1964). Response parameters including synergismantagonism in aqueous odor measurement. Annals of New York Academy of Sciences, 116, 495-503.

Bartoshux, L. M. (1975). Taste mixtures: Is mixture suppression related to compression? Physiology \& Behavior, 14, 643-649.

BERglund, B. (1974). Quantitative and qualitative analysis of industrial odors with human observers. Annals of the New York Academy of Sciences, 237, 35-51.

Berglund, B., Berglund, U., \& Lindvale, T. (1971). On the principle of odor interaction. Acta Psychologica, 35, 255-268.

Berglund, B., Berglund, U., \& Lindvall, T. (1976). Psychological processing of odor mixtures. Psychological Review, 83, 432-441.

Berglund, B., Berglund, U., Lindvall, T., S Svensson, L. T. (1973). A quantitative principle of perceived intensity summation in odor mixtures. Journal of Experimental Psychology, 100, 29-38.

Berglund, B., Olsson, M. J. (1993). Odor-intensity interaction in binary mixtures. Journal of Experimental Psychology: Human Perception \& Performance, 19, 1-13.

CAIN, W. S. (1975), Odor intensity: Mixtures and masking. Chemical Senses \& Flavour, 1, 339-352.

CAIN, W. S., \& DREXLER, M. (1974). Scope and evaluation of odor 
counteraction and masking. Annals of the New York Academy of Sciences, 237, 427-439.

DE WIJK, R. A. (1989). Temporal factors in human olfactory percep tion. Unpublished doctoral dissertation, University of Utrecht.

Ekman, G., Berglund, B., Berglund, U., \& Lindvall, T. (1967). Perceived intensity of odor as a function of time of adaptation. Scandinavian Journal of Psychology, 8, 177-186.

Engen, T. (1982). The perception of adors. New York: Academic Press.

Frijters, J. E. R., \& OUdE Ophuis, P. A. M. (1983). The construction and prediction of psychophysical power functions for the sweet ness of equiratio sugar mixtures. Perception, 12, 753-767.

GREgson, R. A. M. (1980). A model of paradoxical odor mixture perception. Chemical Senses \& Flavour, 5, 257-269.

GREGSON, R. A. M. (1983). The sequential structure of odour mixture component intensity judgments. British Journal of Mathematical Psychology, 36, 132-144.

GREGSON, R. A. M. (1986). Qualitative and aqualitative intensity components of odour mixtures. Chemical Senses, 11, 455-470.

Jellinek, J. S., \& Köster, E. P. (1979). Perceived fragrance complexity and its relationship to familiarity and pleasantness. Journal for the Society of Cosmetical Chemistry, 30, 253-262.

Jellinex, J. S., \& Köster, E. P. (1983). Perceived fragrance complexity and its relationship to familiarity and pleasantness II. Journal for the Society of Cosmetical Chemistry, 34, 83-97.

JoNES, F. N., \& Woskow, M. H. (1964). On the intensity of odor mixtures. Annals of the New York Academy of Sciences, 116, 484-493.

Kendall, D. A., Neilson, A. J. (1966). Sensory and chromatographic analysis of mixtures formulated from pure odorants. Journal of Food Science, 31, 268-274.

KösTER, E. P. (1969). Intensity in mixtures of odorous substances. In C. Pfaffmann (Ed.), Olfaction and taste III (pp. 140-149). New York: Rockefeller University Press.

Köster, E. P., \& MacLeod, P. (1975). Psychophysical and electrophysiological experiments with binary mixtures of acetophenone and eugenol. In D. G. Moulton, A. Turk, \& J. W. Johnston, Jr. (Eds.), Methods in olfactory research (pp. 431-444). London: Academic Press.

LAFFORT, P. (1989). Models for describing intensity interactions in odor mixtures: A reappraisal. In D. G. Laing, W. S. Cain, R. L. McBride, \& B. W. Ache (Eds.). Perception of complex smells and tastes (pp. 173-188). New York: Academic Press.

LAFFORT, P., \& DRAVNIEKS, A. (1982). Several models of suprathreshold quantitative interaction in humans applied to binary, temary and quaternary mixtures. Chemical Senses, 7, 153-174.

LaING, D. G., \& Francis, G. W. (1989). The capacity of humans to identify odors in mixtures. Physiology \& Behavior, 46, 809-814.

Laing, D. G., Panhuber, H., Willcox, M. E., \& Pittman, E. A. (1984). Quality and intensity of binary odor mixtures. Physiology \& Behavior, 33, 309-319.

Laing, D. G., \& Willcox, M. E. (1983). Perception of components in binary odor mixtures. Chemical Senses, 7, 249-264.

LAING, D. G., \& Willcox, M. E. (1987). An investigation of the mechanisms of odor suppression using physical and dichorhinic mixtures. Behavioural Brain Research, 26, 79-87.

LINDVALL, T. (1970). On sensory evaluation of odorous air pollutant intensities. Nordisk Hygienisk Tidskrift (Suppl. 2), 1-181.

Moskowitz, H. R., \& BARBE, C. D. (1977). Profiling of odor components and their mixtures. Sensory Processes, 1, 212-226.

Patte, F., \& LAFfort, P. (1979). An alternative model of olfactory quantitative interaction in binary mixtures. Chemical Senses \& Flavour, 4, 267-274.

Rosen, A. A., Peter, J. B., \& Middleton, F. M. (1962). Odor thresholds for mixed organic chemicals. Joumal of the Water Pollution Control Federation, 34, 7-14.

(Manuscript received November 25, 1991; accepted for publication October 16, 1992.) 